\title{
Developing Open Innovation: Exploring Social Innovation Design in Hong Kong
}

\author{
Helen K. Liu \\ National Taiwan University \\ helenliu4@ntu.edu.tw
}

\author{
Peter Cheung \\ The University of Hong Kong \\ tsyicheu@hku.hk
}

\begin{abstract}
The open innovation model, which allows idea exchange between organizations and the public, is adopted to facilitate social innovation. This study examines the motivational factors and incentives design of open innovation from both the participants and organizations perspectives in the context of Hong Kong. Building upon the existing literature, we investigate how monetary rewards, task meaningfulness, social interaction, and reputation influence the effort invested in and quality of open innovation contributions. We collected survey data on participants' motivation, background and individual characteristics, and their effort and contributions toward open innovation in Hong Kong $(N=155)$. We then built three incentive design models for open innovation development in Hong Kong based on 3 investigated cases to illustrate them. Our findings generate applications for policymakers and implementers who are interested in designing effective open innovation that facilitates social innovation in cities that are going through transitions like Hong Kong.
\end{abstract}

\section{Introduction}

Governments and nonprofits are increasingly utilizing information communication technology (ICT) to facilitate social innovation. Open innovation is "the use of purposive inflows and outflows of knowledge to accelerate internal innovation and expand the markets for external use of innovation" [1]. There has been growing interest in the use of open innovation worldwide as well as in making use of the public's "wisdom" to solve problems that were previously solved internally [2]. For instance, the recent Open Government movements reflect a growing trend of governments experimenting with "outsourcing" government projects to the public, such as in Challenge.org [3]. More importantly, open innovation in the public sector is different in terms of the focus, aim, value, and external stakeholders [4]. For instant, open innovation in the public sector often aims for service improved and the stakeholders of project involve citizens, higher education, nonprofits, governments. Meanwhile, studies have also shown that the main challenges of implementing open innovation include a low participation rate, citizen motivation, and infrastructure design [5]. Thus, studies on open innovation are increasingly focusing on understanding the motivations and incentive designs behind participation and performance $[4,5]$.

One of the key factors to successfully implementing open innovation is understanding what motivates the public to participate and how to design incentives and infrastructure [5, 6, 7]. A "vibrant, committed community", in most cases, is the most important factor in achieving successful open innovation [6]. A former Chief Technology Officer leading the Open Government Initiative has noted that to make the open innovation model effective in the public sector, future research agendas should address the following: (1) who participates, (2) how they participate, and (3) how and what types of motivation and incentives are provided to attract the right stakeholders [8].

While studies have been conducted to investigate the incentives for participation in Western settings, West-East cultural differences have not been sufficiently accounted for. For instance, previous studies have found that solvers who contributed to open innovation in the Chinese private sector were motivated by higher rewards $[9,10]$ and fairness in reward distribution [11]. These findings show that participants in China appear to be motivated by monetary rewards and fairness and are less likely to be influenced by the other types of intrinsic rewards that have been found to be important in the Western context. However, little is known about the reasons people participate in open innovation in the specific context of Hong Kong, which is to some extent an East-West hybrid.

The purposes of this study are to investigate the emerging open innovation practices in Hong Kong in 
order to understand the new invention in a different culture context. To comprehensively understanding these emerging practices on open innovation in a different context, this study first built the framework from two perspectives: (1) participants' motivation and (2) incentive design of open innovation. Then, our research team conducted survey on participants of open innovation and in-depth case studies with open innovation organizers. Next section will discuss the literature framework.

\section{Motivation and Incentives in Open Innovation}

The existing literature has identified and discussed the factors that influence participation in and the performance of open innovation from two perspectives: one focuses on the participants' motivation and are based on survey and experiment methods. Another one investigates the incentive designs of open innovation itself and adopt case method. Section 2.1 will first discuss the types of motivation empirically tested in the literature, and Section 2.2. will discuss the incentive designs of open innovation practices on participants' participation and performance documented in the literature.

\subsection{Motivation for Open Innovation}

Monetary and Other Rewards. Payment is always identified as an important motivational factor driving the number of contributions $[12,13]$ and the amount of time spent engaging with Amazon's Mechanical Turk [14], idea generation in IdeaConnection (Battistella \& Nonino 2012), and creative designs in Threadless.com [12, 13]. Existing empirical evidence shows that monetary rewards are an important factor for increasing participation. However, Garcia Martinez and Walton (2014 argued that increasing the monetary amount of an award would not increase crowd motivation, although it would attract more participants and increase the probability of obtaining a successful solution.

Skill Development \& Task Meaningfulness. Others have found that task characteristics such as task autonomy and skill variety are important for sustaining the contribution levels in task performance projects, such as for Amazon's Mechanical Turk [14], MobileWorks [15] and TaskCN [16]. These studies found that participants made repeated contributions because they found the tasks challenging and felt able to improve their skills by doing the complex tasks assigned to them $[12,13,17]$. Additionally, some participants seek to demonstrate their abilities through certain tasks to attract their future employers, such as the participants in SAPien [18, 19].

Enjoyment. While some people take open innovation projects seriously and treat them as another form of employment, others contribute to innovation projects because they align with their interests, such as the participants in Galaxy Zoo [13], Threadless [12, 13], and the Atizo innovation platform [20]. Some participants enjoy the challenges of solving a problem in addition to the financial reward [17, 21]. Not surprisingly, enjoyment and fun stood out in several research surveys when participants were asked what motivated them to contribute. Open innovation projects that provide a space for people with similar interests and hobbies to meet and interact clearly receive more contributions than those that do not.

Social Interaction. In studying Dell's IdeaStorm, study showed that enabling participants to post comments for others who have different ideas is positively associated with the number of ideas that are selected and implemented [22]. In Galaxy Zoo, participants contribute and stay active to make friends and socialize with others who share their interest in astronomy [13]. Considering the case of Nokia's IdeasProject, it is important for a company to sustain its open innovation initiatives by making active contributors more visible, providing opportunities for interaction within the community and fostering responses from members [23].

Reputation. Social processes play an important role in sustaining the community, complementing monetary incentives. One way of creating social interaction is by building a reputation system [17] and establishing a system of peer recognition, as in the Next Stop Design Project [24]. Participants are, in fact, eager to seek peer recognition and keen to build their reputation by interacting with others on the Internet.

\subsection{Incentives and Designs of Open Innovation}

The Nature of the Open Innovation. The performances of participants are "functions of the goal (communication level; participation level; clarity level); the nature of the tasks (variety; specificity; autonomy and discretion; significance; interdependence); the social structure (hierarchy-neutral; hierarchical) and the nature of the good (public good; private good)" [13]. While platforms like Amazon's Mechanical Turk demonstrate a clear goal, low diversity, hierarchical social structure, private good, and the lack of intrinsic motivation, monetary incentives become the most important factor. On the other hand, Galaxy Zoo and Moon Zoo platforms showed clear goals, low variety and specificity, only trivial skills required, neutral social structure, public good, the participants were 
motivated simply by their interests in astronomy and aided by the capacity of the task dimensions [13]. While platforms like Threadless show clear goals, greater variety, specificity, and identification, highly specific skills required, hierarchical structure with democratic features (i.e. voting), and private good, they find that participants are also motivated by monetary and skills development [13].

Innovation Process. Generating innovations and producing massive and complex tasks requires time, collaboration and commitment, people's motivations would vary according to the phase of the innovation process [25, p.570]. They found that as the innovation process is becoming concrete (such as from foresight to design), the more extrinsic incentives (monetary rewards) are adopted to induce the quality outcomes, such as the case of IdeaConnection [25]. Furthermore, it is important to incentivize activities that you wish to happen, such as user interaction, reviewing, commenting, evaluation, voting, etc. For instance, when comparing information contributions of online idea competition and traditional focus group, study finds that idea competitions lead to more and better ideas at a lower cost per idea while focus group yields richer interactions with users [26]. They argue that when tasks and actions, such as interaction, are not incentivized, users would not perform then [26]. So, it is important to find the right incentives for participant to perform tasks of desired.

\section{Method and Data}

\subsection{Research Design}

The survey instrument was built based on literatures on open innovation motivation and incentive design [12, 18, 20, 27]. Demographic items such as age, education, computer skills, and social media usage and experiences in open innovation are also included in the survey. The survey instrument of our study measures whether different types of motivation and incentives designs might affect the participation in the open innovation activities in Hong Kong. We also include open-ended items, so that the incentives will not be limited by the cultural settings or age groups in which survey were conducted in previous literature. Specifically, previous surveys asked the participants to rate the scale of importance of each motivational factor by using 5-point Likert scales [12, 18, 19, 20]. Similarly, we will adopt 5-point Likert scales (where 1 $=$ the lowest agreement, $5=$ the strongest agreement) to rate the importance of each motivational factor. The average time to complete the survey is about 30 minutes. In previous experiences of surveying participants, the average response rate has been about
$30 \%$ [24], and the response rate of our study was approximately $84.5 \%$.

For our case study on incentive designs, following conceptual studies [28, 29], the survey instrument used in our study measures whether and to what extent the incentives motivate participation in the open innovation setting in Hong Kong. Each interview with the organizations lasted for approximately 40 to 60 minutes and covered questions regarding program objectives, criteria, performance, social impact, challenges and policy recommendations with reference to their respective open innovation programs.

\subsection{Data Collection}

This study built on both Braham's [24] and Budhathoki and Haythornthwaite's [27] studies by conducting interviews with previous participants in open innovation initiatives in Hong Kong. To reduce the potential bias in comparing the outcomes of open innovation platforms, we selected open innovation projects that were launched or have been in operation for at least one year, including Carbon Care Innolab, DreamCatchers HKU, Good Seed and Youth Connect MTR in Hong Kong. This will minimize variations in intrinsic motivations that arise as a result of networks and social relations that have been fostered as a function of time.

Then, we were able to recruit interviewees from the past participant pool in the selected open innovation platforms for our survey interviews. We recruited and interviewed interviewees directly from the indicated open innovation events in Hong Kong during summer 2017 through fall 2017. Some have rich experience in developing open innovation programs with social impacts while some are still in the pilot phase. To control for the influence from experiences, we also interviewed university students who had learned about but lacked participation experience, as we aimed to compare the results for people with different levels of participation in open innovation in later phase of our research.

In sum, the research team conducted face-to-face interviews with 155 individuals who attended the three selected open innovation related events, including training workshops and competitions; these interviews allow us to understand their experiences, motivation and preferences in incentive designs. Additionally, to learn about the design of open innovation, we further interviewed 3 nonprofit organizations and 2 open innovation winners, and these results in three case studies to further understand the nature of the open innovation and innovation process designed by different organizers. 


\subsection{Open Innovation in Hong Kong}

Open innovation is an emerging concept and often occurs in the business and information technology sector in Hong Kong [30]. Hong Kong was ranked 16th in the Global Innovation Index 2017 out of the 127 economies surveyed, with very strong performances in knowledge and creative outputs [31]. With its international financial center status and talent pool, Hong Kong has a relatively solid foundation to further development open innovation.

In the public and nonprofit sector, most new initiatives are organized by private foundations, nonprofit organizations and universities while working closely with governments as sponsored organizations. One of the pioneering efforts in adopting open innovation for social purposes in Hong Kong was the Hong Kong Social Enterprise Challenge, a social venture startup competition launched in 2007 and organized by the Chinese University of Hong Kong. Since then, similar initiatives have emerged in both tertiary education and commercial realms around Hong Kong, including Carbon Care Innolab, DreamCatchers HKU, Good Seed and Youth Connect MTR. More recently, the French Chamber organized the Open Innovation Forum in partnership with Agorize in April 2018, which aims to match start-ups with the innovation ecosystem.

However, the advancement of open innovation in the public and nonprofit sector in Hong Kong is facing considerable challenges. First, the concept of tapping into the wisdom of the crowds to resolve public problems is relatively novel in Hong Kong; this indirectly leads to problems locating appropriate participants, gaining trust from the founders, and obtaining quality responses. Second, open innovation is generally not perceived as career development in Hong Kong, which impedes the willingness of trying from the young generation. Third, the resources dedicated to the implementation of the ideas generated as a result of open innovation are often insufficient to sustain their operation. Fourth, the initiatives tend to face difficulties identifying and understanding the motivations behind participation, rendering the design of the challenges less effective at attractive the ideal rate of participation.

\section{Findings from the Participant Perspectives}

\subsection{Characteristics and Experiences of the Participants}

Our study reveals that $29.67 \%$ of respondents found that the open innovation challenges they participated in were within of their expertise. While $24.18 \%$ of respondents indicated that the challenges they participated in were closely related but outside their field of expertise, $23.08 \%$ of respondents indicated that the challenges participated in were slightly different but remained within their field of expertise.

The submissions of approximately 52\% of respondents were based on a solution that they had developed in their own work, while the other $48 \%$ of respondents based their submissions on an existing solution they knew about that could solve the open innovation challenge.

Our participants ranged from having professional experience to having no experience at all in the topic of the challenge. Most commonly, participants had experience with the problems presented in the challenges as a student, with approximately 35\% of respondents agreeing with such a statement. Approximately $33 \%$ of respondents indicated that they had experience with the problems presented as a hobby, and approximately $32 \%$ of respondents indicated that they had experience with the problems presented professionally.

According to our survey, $4 \%$ of the respondents were satisfied with their experience with the open innovation challenge they participated in, $48.21 \%$ of them had no comment and the remaining $18.75 \%$ were dissatisfied. However, most of the respondents stated that they might be or would be willing to attempt an open innovation challenge in the future (98.46\%), despite the high level of dissatisfaction.

The respondents further indicated their inclination toward future participation in open innovation challenges. Approximately 54\% of the respondents suggested that they would participate in such challenges again in the future, and approximately $44 \%$ of the respondents stated that they might participate. Only approximately $2 \%$ of the respondents gave a negative answer regarding any future participation.

Based on the participation experiences from our interviews, we identified four types of participants based on their participation patterns: prize hunters, innovators, conservatives, and second career seekers (Figure 1). Prize hunters are those who participate in multiple contests aiming to win prizes to sustain their startup. Innovators are those who have participated multiple contests and won the idea competitions. Conservatives are those who hesitant to contribute their ideas, even though they have participated in discussions, training workshops and have some degree of knowledge of open innovation. Second career seekers treat the open innovation event as an actual career; they are often middle-aged men and women who seek alternative career paths. 
Types of Open Innovation Participants

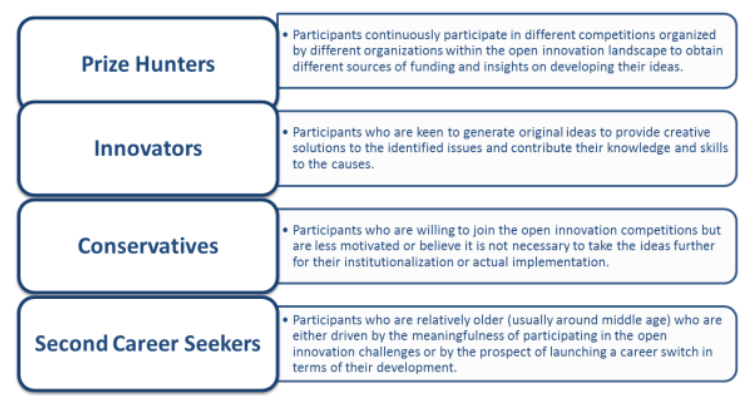

Figure 1 Types of Open Innovation Participants

\subsection{Motivation}

Monetary is not essential. According to our survey, monetary reward may not be necessary to attract participation in open innovation challenges because the majority of respondents might $(48.46 \%)$ or would (33.84\%) have attempted the challenge without a financial reward. Previous research shows that open innovation participants in China appear to be motivated by monetary rewards and are less likely to be influenced by the other intrinsic rewards that have been found to be important in the Western context. The findings from our Hong Kong study do not support the previous findings in China even through both societies are Chinese-based. Instead, it finds that while the monetary reward may still be an important motivational factor, it is not a necessary factor for attracting participation. In additional to the culture different between Hong Kong and China, the differences in findings also might be a result of the type of open innovation that we have selected. Previous studies focused on open innovation development in the private sector in China where our study in Hong Kong focused on social innovation.

Skill Development and Task Meaningfulness are essential. The responses of the participants indicated that the major motivational factors leading them to participate in open innovation programs concern the learning process and the meaningfulness of the tasks. In our survey of reasons for participating in open innovation, our interviewees reported three of the top five reasons were (1) to enhance their skills (3.73), (2) to enhance their career prospects (3.65), and (3) to learn about these types of challenges (3.4). It was also found that while the open innovation challenges most participants participated in were at the boundary of their field of expertise, most open innovation programs offered training and workshops at the preliminary stage to enable participants to acquire the requisite skills and knowledge before taking part in the challenge.
Enjoyment is also essential. Our interviewees reported two of the top five reasons were motived by the enjoyment: (1) because they enjoy solving these types of challenges (3.47) and, and (2) for an intellectual challenge (3.19). Also,

Social interaction is not a motivator, but a necessary skill. While our interviewees didn't rank social interaction as one of the top five motivators, approximately $88.75 \%$ of respondents solved the open innovation challenge as a team, while the remaining $11 \%$ of respondents worked individually $(11.25 \%)$. Team participation is a key characteristic of the open innovation programs we have studied because one of the key objectives of these programs is to offer the opportunity for participants to engage in multidisciplinary collaboration.

\subsection{Preferences on Incentive Designs}

The Nature of the Open Innovation. Our finding support Tokarchuk et al [13] study as our survey show that the nature of the open innovation matters the most. For instance, on the design factors that attract participants, the most important factor is (1) "my response can make a difference in the outcome" (3.87). The findings show that participants care about the purpose of the open innovation programs they participate in. As observed in the participant interviews, they consider the social impact of their submissions to be a key motivational factor. They want to see their ideas implemented and to help provide solutions to social problems. This is consistent with the findings in the existing literature. Existing studies show that the meaningfulness of the tasks (Chandler \& Kapelner, 2013) and feedback from the organizations (Bayus, 2013) are key to sustaining the community. Participants are more likely to participate in future challenges if they find meaning in their contribution even though they may not win the final prize. Additionally, studies show that feedback from peers and the organizations is also an essential reason for participants to return to challenges [8].

Innovation Process. As mentioned earlier, generating innovations requires time, collaboration and commitment, people's motivations might vary according to the phase of the innovation process. For instance, we find that during the initial stage of attracting participants, the design of the program, including the flexibility, transparency and ease of use of the platform, is also important. As many open innovation programs involve the use of online platforms to facilitate participation and submission, an easy and user-friendly platform will enable participation. As Wang (2008) shows, the easy access, 
flexibility and ease of use of the platform are key initial requirements to encourage participants to post and participate on the online platform or discussion forum.

Furthermore, during the idea submission stages, we find that there were several factors that might discourage participants from submitting ideas: (1) "unattractive rewards" (2.8), (2) "no interest" (2.79), (3) "poor computer skills" (2.77), (4) "unfamiliar with the social issue/policy" (2.74), and (5) "no new ideas and no spare time" (2.68). Our interviews show that other forms of reward, such as further support after winning the competition and the learning experience involved, also play a role. Also, we learned that lack of ability and capacity are key factors discouraging participants from trying to share their ideas. Thus, training workshops or idea brainstorming sessions are essential to increase the confidence levels of participants and encourage them to submit their ideas.

Finally, for any form of participation, such as commenting or reviewing, we also find some concerns from the participants: (1) reliability of information (36.1\%), (2) poor computer skills $(33.0 \%)$, (3) security of personal information $(30.9 \%)$, (4) online accessibility (19.6\%), and (5) peer pressure (14.4\%). From our survey, concerns about online security and the reliability of the online information may deter participation even for just commenting or reviewing of ideas.

\section{Open Innovation Incentive Designs and Emerging Models}

\subsection{CarbonCare InnoLab}

Carbon Care InnoLab (CCIL) was founded by the Jockey Club in 2015 to generate innovative solutions addressing climate change and environmental protection. The JC CCIL is an 8-month incubation program that provides intensive coaching to help participants realize their green projects. Events are held throughout the program to allow participants to exchange ideas and form groups for open innovation competition, where winners can receive a startup fund to establish their business. The program is held annually, and the first competition was held in 2016.

The innovation process involved multiple steps. Step 1 included CarbonCare InnoFest, which allowed the potential participants to learn about the innovation, business and environmental protection concepts as well as to meet potential group members for later workshops. Step 2 included a bootcamp that allowed participants to form groups and work on their startup ideas. During Step 3, early training included six teams that were shortlisted and received training on the Lean Startup methodology. Step 4 was the qualifying pitch, and the six teams presented proposals to be reviewed by JC CCIL. Four winning teams received a grant of $\mathrm{HK} \$ 8000$ each to proceed to the next stage. For Step 5, project idea testing, the four teams could conduct market analysis and test users' response to their projects. Finally, in Step 6, the four teams compete for the final award of up to $\mathrm{HK} \$ 50000$ by presenting their ideas before a panel of judges.

The program director of JC CCIL stated that the essential design for an effective open innovation was to involve diverse backgrounds. More importantly, through her personal observation and experiences, she found the meaningfulness of the competition was a key incentive to attract the real talents. The program director of JC CCIL, Cheng, stated that the diverse backgrounds of participants contribute to more comprehensive viewpoints and ideas in the process of developing the projects and discussing issues.

However, the biggest challenges were the recruitment of participants and the identification of effective ideas. As open innovation is still new in Hong Kong and the concept of CCIL is not very concrete and specific, it is difficult to attract potential participants. Because open innovation is not yet common or popular in Hong Kong, they find that only a small community of participants is interested in contributing to open innovation, which makes it hard to reach more diversified groups.

\subsection{Hong Kong Social Enterprise Challenge}

Established in 2007, the Hong Kong Social Enterprise Challenge (HKSEC) is a social venture startup competition that is organized by the Center of Entrepreneurship at the Chinese University of Hong Kong, with sponsorship by the Home Affairs Bureau. HKSEC aims to foster innovative and practical social enterprise ideas by engaging multidisciplinary and multi-institutional teams. The main aim of the program is to faculiate ideas from the private and nonprofit sectors to address public and social problems.

Social venture plans can address local, national, regional or global social needs, which may include problems faced by new immigrants, senior citizens or patients. The plan should address a social problem, e.g., healthcare, education, the environment or social services. Furthermore, the program not only call for idea proposal, but also request the team to propose the design of the organization. The proposed organization should contain at least the double bottom line, which is a sustainable financial return and a distinguished social mission, although environmental neutrality as the third bottom line is always welcome. 
With a strong aim of discovering social innovators, the HKSEC is open to students enrolled in postsecondary programs at universities and colleges in Hong Kong, including overseas graduates with Hong Kong citizenship and recent graduates. The HKSEC also invited judges, who play a key role in deciding which teams advance to the semifinal and final rounds of the challenge. The panel of judges includes executives and NGOs, and their decision is based on HKSEC's judging criteria, namely, concept, context, social impact, sustainability, and people. Furthermore, faculty advisors are invited to supervise students and are composed of professors and lecturers.

The championship teams will receive HKD $\$ 60,000$ in startup prize money to implement the first phase of their business plan for their social enterprise under mentorship. By 2017, HKSEC had successfully established 30 social enterprises and has gradually evolved to become a hub providing social innovations. In the past 10 years, HKSEC has attracted 6762 participants with 1166 business plans. Winning projects include Isee Mobile Apps, HEYCOIN, Eco Tour, etc.

\subsection{MTR Youth Connect}

Youth Connect is another project that comprises a series of programs organized by the MTR Corporation. With the goals of empowering young people with skills, motivation and new perspectives and building a sustainable future, Youth Connect provides different initiatives for youth.

Youth Connect, began as "Pathways to Employment", is an open innovation program that involved online and offline engagements. The program started with a Cross-Sector Summit that brought together over 300 stakeholders - young people as well as representatives from the business, education, and NGO sectors - to deepen their understanding of the challenges in the path from education to work. The Summit resulted in agreement that there is a need for greater cross-sectorial collaboration to build effective bridges for youth employment, and in response, the MTR Corporation launched an online Community Innovation Platform to facilitate the development of solutions that address young people's needs.

Through the application of design thinking, an innovation process that combines creative and critical thinking skills to solve problems in a user-centric way, MTR leveraged the knowledge and expertise in the community to develop innovative and creative solutions. MTR funded five projects that were created by and chosen by the community. These initiatives focus on creating the next generation of game changers and entrepreneurs, enriching the city's education system, and empowering girls to take up technology subjects. The uniqueness of the program lies in the design-thinking process, which allows ideation and the refining of ideas on an online platform, which encourages participants to build on each other's ideas and support each other. It should be noted, however, that one main challenge was getting people online to share their ideas, as the concept of competing for resources stopped some NGOs from participating in this highly transparent platform.

In terms of motivation, the project manager of the Youth Connect identified funding support as the major driver of the program. Some NGOs said that "they struggled to find funders in the first place, and when they do find it, there are lots of conditions. We were more relaxed in the process", reported the project manager. To help people keep an eye on the upcoming events, the preliminary communication and "preevents" are necessary. Additionally, the online platform needs to be user-friendly and designed based on the participants' experience to ensure that all the participants, especially older participants, are able to navigate the platform easily.

Other programs under Youth Connect include Pathways to Employment, STEM Challenge, 'Train' for life's journeys, Customer Service Ambassadors and Tourist Ambassadors Internships, and 'Life skills training - Hong Kong Athletes Career and Education Programme'. Through this range of programs, MTR supports 7,500 young people every year. The program provides additional infrastructure to encourage young people to create their own businesses; this includes improving their competence in working with consumer markets. The initiatives not only work to bolster confidence and broaden perspectives but also help learners nurture soft skills, develop creative thinking, and generate networks that can benefit them in their careers.

In one interview, Senior Manager of Corporate Responsibility, shared the impact of Youth Connect, discussing how it benefited young people by providing a better education and a better learning environment, and how it benefited NGOs by teaching them how to better design their projects through a toolkit that adopted design-thinking principles to support project planning and implementation processes.

\section{Discussions on Open Innovation Models}

The case studies illustrate common practices of open innovation adoptions in Hong Kong for developing social innovations in the public and nonprofit sector. In particular, all three cases demonstrate three idea development stages, namely 
idea generation, idea selection, and idea implementation (Figure 2). During the idea generation stage, our interviewees reported that idea brainstorming and workshops for skills and knowledge development were commonly adopted. During the idea selection stage, a peer review or a panel of judges for ideas selection would be adopted during the challenges or idea competitions. Finally, in the idea implementation stage, our interviewees reported that their organizations would help the idea winners to set up their start-up or institution and provide guideline of market entry or connection to the industry. However, while all three cases design their challenges based on these three stages, but most of them focus on idea generation and selection. Our interviewees expressed the needs for further development on idea implementation.
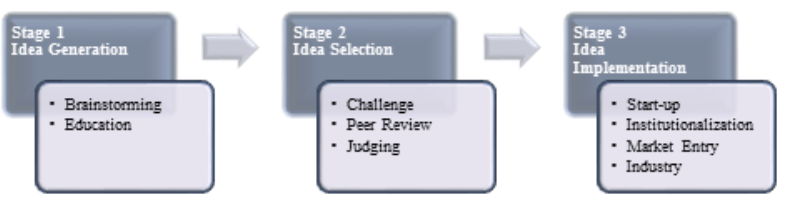

Figure 2 Three Stages of Open Innovation in Hong Kong

Furthermore, as mentioned in our literature review about the importance of innovation process, the case studies show four components of the open innovation models in Hong Kong (Figure 3). Education refers to the role of organizations assume in nurturing incubatees by providing suitable and timely guidance and assistance to support the generation, design and experimentation with ideas. Innovation refers to the role of organizations in incentivizing the creation of ideas and weighing their merits and effectiveness in resolving the defined issues. Fund generation refers to the role of organizations in obtaining sustainable and continuous sources of funding to provide sufficient financial support to incubatees for the realization and institutionalization of their ideas. Promotion refers to the role of organizations in fostering an open innovation culture and promoting it to a broader audience beyond the incubatees to create a vibrant open innovation landscape. The four roles are to be viewed as a process within which open innovation challenges are developed.

\section{Open Innovation Models}

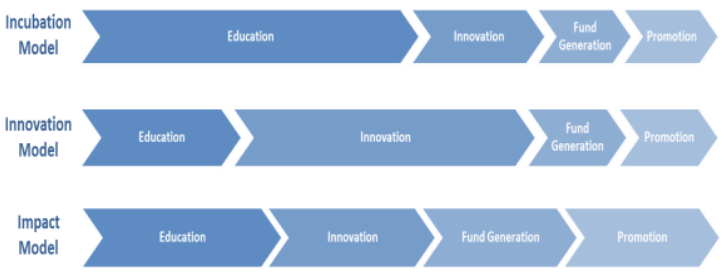

Figure 3 Open Innovation Models in Hong Kong

Our interview and case studies reveal three open innovation models in Hong Kong (Figure 3). The first model is an incubation model. We classify CCIL as an incubation model that incorporated training-intensive workshops with the aim of discovering and cultivating new participants with passion and knowledge about environmental issues. The second model is an innovation model. We classify HKSEC as an innovation model that included design thinking or solution-based methods with the aim of generating innovations to solve problems of interest. The third model is an impact model. We identify YC as an impact model that builds the capacity of its participants and further seeks funds for them to implement their proposals and innovation.

\section{Conclusions}

Advancements in technology have enhanced the ability of government and nonprofit organizations to solicit ideas and help support social innovation. Our findings show that monetary rewards are not necessary to attract participation in open innovation in Hong Kong because the findings show that most respondents would have attempted open innovation even if no financial reward had been offered. Instead, the underlying factors motivating participation are skill enhancement, the improvement of career prospects, learning opportunities and intellectual exchange. In addition, it is found that social impact and the program design of the particular open innovation challenge, including flexibility, transparency and ease of platform use, are important to facilitate open innovation. Meanwhile, online security and accessibility are potential obstacles that deter participation in online open innovation.

When examining the incentive and model designs, the open innovation ecosystem in Hong Kong is reported to be fragmented and unsystematic, mainly because Hong Kong lacks an enabling environment, including a proper regulatory framework and the availability of angel investors, both of which can 
facilitate and encourage the development of open innovation. As suggested by the open innovation organizers in Hong Kong, information sharing and cooperation among different organizers of open innovation are not common. For instance, from our interviews, several participants repeatedly joined different open innovation programs, known as the "prize hunters," in order to maintain their funding after winning the initial start-up fund. However, this approach is not sustainable and reduce the opportunities for the new comers. It is thus important to develop additional funding or resources for idea winners to implement their ideas.

Meanwhile, another reported challenge is a lack of data law in Hong Kong, which restricts the use and sharing of information. Under the Personal Data (Privacy) Ordinance, which strictly regulates data privacy, sharing personal data and information is difficult. While privacy rights should be protected, interviewees mentioned the importance of accessing public data to facilitate the free flow of information and big data analysis, which is conducive to open innovation. Rethinking a new regulatory framework for data privacy and proposing open data laws are pressing needs to enable the more prosperous development of open innovation.

Recommendations were made regarding the creation of an open culture in the public and nonprofit sector in terms of users, data and value creation [32]: (1) for users, the cultivation of relationships between the government and users to concrete information based on existing data; (2) for data, the development of an infrastructures for data creation and governance; (3) for value creation, documentation of "who benefits and how valued is generated" (p.922). These recommendations are also applicable to building open cultures in Hong Kong to sustain the existing open innovation models revealed in our study.

While different organizations adopt different approaches to developing open innovation, active collaboration among them is necessary to build a balanced and comprehensive ecosystem. From our interviews, the present collaboration is mostly limited to the promotion level. It is crucial to promote active collaboration and idea exchanges across different organizations because it can maximize the outcomes of idea exchanges with the least amount of effort and resources. Thus, incubators should consider building a shared network with digital enablers such as big data and online platforms and physical enablers such as joint events and physical workspace to allow greater accessibility and the more efficient use of resources for incubatees within the ecosystem.

\section{Acknowledgement}

This research project (Project Number: 2016.A8.052.17A) is funded by the Public Policy Research Funding Scheme from Policy Innovation and Co-ordination Office of the Hong Kong Special Administrative Region Government.

\section{References}

[1] Chesbrough, H. W. "Open innovation: The new imperative for creating and profiting from technology", Boston, MA: Harvard Business School Press, 2006

[2 ] Dixon, B. E. "Towards e-government 2.0: An assessment of where e-government 2.0 is and where it is headed", Public Administration \& Management, 2010, 15(2), 418-454.

[3] Mergel, I., \& Desouza, K. C. "Implementing open innovation in the public sector: The case of Challenge.gov." Public Administration Review, 2013, 73(6), 882-890. doi:10.1111/puar.12141.

[4] Kankanhalli, Atreyi, Anneke Zuiderwijk, and Giri Kumar Tayi. "Open innovation in the public sector: A research agenda." (2017): 84-89.

[5] Gascó, Mila. "Living labs: Implementing open innovation in the public sector." Government Information Quarterly 34, no. 1 (2017): 90-98.

[6] Howe, J. "The rise of crowdsourcing", Wired, 2006, $14(6), 1-4$.

[7] Afuah, A., \& Tucci, C. L. "Crowdsourcing as a solution to distant search", Academy of Management Review, 2012, 37(3), 355-375. doi:10.5465/amr.2010.0146.

[8] Noveck, B. S. Wiki government: How technology can make government better, democracy stronger, and citizens more powerful, Washington, DC: Brookings Institution Press. 2009.

[9] Liu, T. X., Yang, J., Adamic, L. A., Chen, Y. "Crowdsourcing with all-pay auctions : A field experiment on TaskCN", Management Science, 2014, 60(8), 2020-2037. doi:10.1287/mnsc.2013.1845.

[10] Shao, B., Shi, L., Xu, B., \& Liu, L. "Factors affecting participation of solvers in crowdsourcing: An empirical study from China", Electronic Markets, 2012, 22(2), 73-82. doi:10.1007/s12525-012-00933.

[11] Zou, L., Zhang, J., \& Liu, W. "Perceived justice and creativity in crowdsourcing communities: Empirical evidence from China", Social Science Information, 2015, 54(3), 253-279. doi:10.1177/0539018415583382.

[12] Brabham, D. C. "Moving the crowd at Threadless. Information", Communication \& Society, 2010, 13(8), 1122-1145. doi:10.1080/13691181003624090.

[13] Tokarchuk, O., Cuel, R., \& Zamarian, M. “Analyzing crowd labor and designing incentives for humans 
in the loop", IEEE Internet Computing, 2012, 16(5), 45-51. doi:10.1109/MIC.2012.66.

[14] Kaufmann, N., Schulze, T., \& Veit, D. "More than fun and money: Worker motivation in crowdsourcing - A study on Mechanical Turk", AMCIS, 2011, Proceedings.

[15] Pilz, D., \& Gewald, H. "Does money matter? Motivational factors for participation in paid-and non-profit-crowdsourcing communities", Wirtschaftsinformatik, 2013, 37.

[16] Zheng, H., Li, D., \& Hou, W. "Task Design, Motivation, and Participation in Crowdsourcing Contests", International Journal of Electronic Commerce, 2011, 15(4), 57-88. doi:10.2753/JEC10864415150402.

[17] Marjanovic, S., Fry, C., \& Chataway, J. "Crowdsourcing based business models: In search of evidence for innovation 2.0", Science and Public Policy, 2012, 39(3), 318-332. doi:10.1093/scipol/scs009.

[18] Ebner, W., Leimeister, J. M., \& Krcmar, H. "Community engineering for innovations: The ideas competition as a method to nurture a virtual community for innovations", R\&D Management, 2009, 39(4), 342-356. doi:10.1111/j.14679310.2009.00564.x.

[19] Leimeister, J. M., Huber, M., Bretschneider, U., \& Krcmar, H. "Leveraging crowdsourcing: Activation-supporting components for IT-based ideas competition", Journal of Management Information Systems, 2009, 26(1), 197-224. doi:10.2753/mis0742-122226010

[20] Frey, K., Lüthje, C., \& Haag, S. "Whom should firms attract to open innovation platforms? The role of knowledge diversity and motivation", Long Range Planning, 2011, 44(5-6), 397-420. doi:10.1016/j.lrp.2011.09.006.

[21] Lakhani, K. R., Jeppesen, L. B., Lohse, P. A., \& Panetta, J. A. "The value of openess in scientific problem solving", Division of Research, Harvard Business School, pp. 07-50.

[22] Bayus, B. L. "Crowdsourcing new product ideas over time: An analysis of the Dell IdeaStorm community," Management Science, 2013, 59(1), 226-244. doi:10.1287/mnsc.1120.1599.

[23] Kosonen, M., Gan, C., Vanhala, M., \& Blomqvist, K. "User motivation and knowledge sharing in idea crowdsourcing", International Journal of Innovation Management, 2014, 18(5). doi:10.1142/S1363919614500315.

[24] Brabham, D. C. "Motivations for participation in a crowdsourcing application to improve public engagement in transit planning", Journal of Applied Communication Research, 2012, 40(3), 307-328. doi:10.1080/00909882.2012.693940.

[25] Battistella, C., \& Nonino, F. "Open innovation webbased platforms: The impact of different forms of motivation on collaboration", Innovation, 2012, 14(4), 557-575. doi:10.5172/impp.2012.14.4.557.

[26] Schweitzer, Fiona Maria, et al. "Crowdsourcing: Leveraging innovation through online idea competitions." Research-Technology Management 55.3 (2012): 32-38.

[27] Budhathoki, Nama R., and Caroline Haythornthwaite. "Motivation for open collaboration: Crowd and community models and the case of OpenStreetMap." American Behavioral Scientist 57.5 (2013): 548-575.

[28] Jeppesen, L. B., Lakhani, K. R., Lohse, P. A., \& Panetta, J. A. The value of openness in scientific problem solving. 2007.

[29] Liu, H. K. "Crowdsourcing government: Lessons from multiple disciplines", Public Administration Review, 2017, 77(5), 656-667. doi:10.1111/puar.12808.

[30] Yan, Xu, and Calvin Chun Yu. "Strengths and weaknesses of Hong Kong's technology and innovation industry with reference to the extended open innovation model." Journal of Science and Technology Policy in China 4.3 (2013): 180-194.

[31] INSEAD. "Global Innovation Index Report 2017", Cornell University, INSEAD business school and the United Nations'. World Intellectual Property Organisation, 2017, Retrieved from https://www.globalinnovationindex.org/gii-2017report.

[32] Harrison, T. M., Pardo, T. A., \& Cook, M. "Creating open government ecosystems: A Research and Development agenda”, Future Internet, 2012, 4(4), 900-928. doi:10.3390/fi4040900. 